\title{
Output Feedback Recursive Dynamic Surface Control with Antiwindup Compensation
}

\author{
Guofa Sun $\left(\mathbb{D}\right.$, Hui Du $\mathbb{D}^{D}$, Gang Wang $\mathbb{D}^{\mathbb{D}}$, and Hanbo Yu \\ Department of College of Information and Control Engineering, Qingdao University of Technology, Qingdao 266520, China \\ Correspondence should be addressed to Guofa Sun; sunguofa_bo@163.com
}

Received 14 August 2020; Revised 26 November 2020; Accepted 22 January 2021; Published 8 February 2021

Academic Editor: Shubo Wang

Copyright ( $) 2021$ Guofa Sun et al. This is an open access article distributed under the Creative Commons Attribution License, which permits unrestricted use, distribution, and reproduction in any medium, provided the original work is properly cited.

\begin{abstract}
Actuator saturation phenomenon often exists in the actual control system, which could destroy the closed-loop performance of the system and even lead to unstable behavior. Our main contribution is to provide an antiwindup recursive dynamic surface control (RDSC) for a discrete-time system with an unknown state and actuator saturation. The fuzzy compensator is added to perform as an active disturbance rejection term in the feedforward path to avoid windup caused by input saturation. To construct output feedback control, the system is transformed into the form of pure-feedback and an improved HOSM observer is designed to carry out future output prediction. Based on which RDSC is synthesized, only one fuzzy logic system (FLS) is used and the controller singularity is completely avoided. In addition, the simulation and numeral examples using a continuous stirred tank reactors (CSTRs) system with actuator saturation are provided and the results show that the strategy owns good robustness and effectively compensates for the disturbance caused by actuator saturation in the presence of a discrete-time system with an unmeasurable state.
\end{abstract}

\section{Introduction}

Considering that actuators in the actual system usually have an inherent physical input limitation, a further increase of the control signal will not lead to a faster response of the controlled system. This phenomenon will greatly deteriorate the output performance of the system, produce unacceptable errors, and even cause instability of the system. For example, the jumping robot system is composed of a rotatable leg drive structure, which usually needs to be restrained during operation to avoid structural damage. The servomotor platform also has the same problem, which needs to constrain the actuator to ensure the safety of driving. The problem of input saturation has been studied by many researchers [1-4]. Rehman et al. [1] investigated antiwindup problems for nonlinear multiagent systems subject to actuator saturation; the sector-based approach is designed to maximize the region of stability and ensuring the operation of a control signal in the nonlinear domain. However, Zuo et al. [2] designed an antiwindup compensation for the stability and performance degradation of saturated systems and discussed a codesign technique about calculating the antiwindup gain. In order to solve the problems of actuator saturation in practical engineering, there is a huge amount of research literature that have adopted saturation compensation and saturation suppression, for example, jelastic joint robot limitation [5], oversaturation of wind turbines [6], and reactant concentration in the reactor [7]. Input saturation widely exists in the control system; extra effort should be made to avoid windup for improving output control performance.

In some recently reported works, many adaptive controllers have been developed for compensating actuator nonlinearity, including deadzone, backlash, and hysteresis [8-10]. For instance, Dinh [8] introduced a mathematical model for backlash hysteresis compensation about wearable exosuit for upper limb assistance, which could accurately track the reference signal by compensating for time-varying backlash and continuously updating the model parameters. Then, Turner and Kerr [9] proposed a nonlinear modification which can be retrofitted to a dynamic antiwindup compensator. This paper will derive an adaptive dynamic surface control scheme for compensating windup caused by actuator saturation. 
In view of the above problems, some progress has been made in the research of dynamic surface control. Zhang [11] developed multidimensional Taylor network (MTN) to approach the unknown function, and MTN dynamic surface control (MTN-DSC) is proposed for a class of strict-feedback uncertain nonlinear systems with unmodeled dynamics and output constraint. Then, Liu et al. [12] presented a robust control design using the neural network (NN) and DSC method for path following of underactuated surface vessels with input saturation. Similarly, Xin et al. [13] investigated a dynamic surface control for nonlinear multiinput multioutput (MIMO) system based on FLS, which effectively reduced online computation in two cascaded continuous stirred tank reactors (CSTRs).

Implementation of adaptive DSC obtained by backstepping requires the analytic calculation of the partial derivatives of virtual control signals. In [14], Yu et al. presented a new implementation approach for adaptive backstepping control, which used the filtering methods to produce certain command signals and their derivatives which eliminate the requirement of analytic differentiation, simplifying the controller design. In order to further improve the quality of the filter and avoid damaging the stability of the system, the position tracking controller module is developed based on the modified DSC method by using the second-order nonlinear tracking differentiator (NLTD) instead of the first-order filter at each step of the virtual inputs in [15]. In [16], Wang et al. proposed a new sliding mode control method and applied it to the motor drive system such that a prior knowledge on the bounds of uncertainties is not required in the controller design. In [17], Chen et al. included the periodic nonparametric part in the unknown expected control input and established a fully saturated repetitive learning law with continuous switching function to ensure that the estimation of the unknown expected control input is continuous and limited in a predetermined region, so as to ensure higher steady-state tracking accuracy.

On the other hand, for continuous-time systems, in the aforementioned works has been carried out on DCS with antiwindup compensation. In contrast to the large amount of work on nonlinear systems with actuator saturation, only a few results are available in adaptive DCS of discrete-time systems, because discrete-time nonlinear systems are more difficult to deal with and many continuous control schemes are not suitable for discrete-time systems. For example, to solve the problem of "explosion of complexity" in the process of the backstepping method, the noncausal problem of the system should be solved in the discrete-time system first. Noncausal problems are solved mainly by changing the system model [18-20], which further increased the complexity of the controller. Therefore, Zhang et al. [19] applied dynamic surface control based on the filter to simplify the complexity of the algorithm, which resolved the problems of explosion of complexity and noncausal in discrete-time systems caused by backstepping. Then, Liu and Tong [20] presented an adaptive fuzzy controller design for uncertain nonlinear systems with the discrete-time form in a triangular structure and including backlash and external disturbance.

But the control schemes mentioned above are based on the conditions with known states. In practical engineering, due to the limitation of sensors, it is impossible to observe all states of the system, so it is not completely satisfactory to design a controller under the conditions with known states. For discrete-time nonlinear systems with parametric uncertainties, there has been a lot of research on fuzzy or neural network state observers in recent years [21-25]. However, in the aforementioned works, the observer was employed to obtain unknown states while the observer performance to the closed-loop system remains undiscussed.

The main contributions of this paper are summarized as follows:

(1) To mitigate the windup problem caused by input saturation nonlinearity in the discrete-time system, the whole closed-loop control system is analyzed in detail first. Then, input saturation nonlinearity is described by linear part and disturbance term, which is combined with uncertain system functions to compose the total disturbance that affect the control performance. Then, the fuzzy control object is established, where using control signals to avoid the windup of actuator winding is the key issue.

(2) By designing an improved HOSM observer, the stable chattering problem of HOSM is effectively solved and output feedback tracking control is achieved under the assumption that system states are unknown. Since most of the practical systems are states unknown, the proposed adaptive sliding surface algorithm is superior to the existed state-feedback control techniques in control nonlinear systems with input saturation in application. In this design, the improved HOSM observer is developed by a coordinate transformation.

(3) An antiwindup RDSC with fuzzy compensation is derived by the Lyapunov stability method based on filtered command and HOSM observer simplifying the complexity of the algorithm, which resolved the problems of the explosion of complexity and noncausal in discrete-time systems caused by backstepping. Compared to the traditional sliding mode (surface) controller (SMC), the chattering phenomenon is effectively avoided. Finally, the tracking errors of the closed-loop system, which can be made arbitrarily small by adjusting design parameters, are proved to be bounded by performance function.

This paper is organized as follows: Section 2 presents the mathematical model of saturation nonlinearity and nonlinear system. Section 3 gives the fuzzy DSC design and stability analysis of the whole closed-loop system. Section 4 provides a simulation example to validate the effectiveness of the proposed control scheme. Finally, Section 5 concludes this paper.

\section{Problem Statement}

In this Section, saturation nonlinearity is introduced first. Subsequently, a nonlinear plant in a triangular form is given for analysis and the control objective is established. 


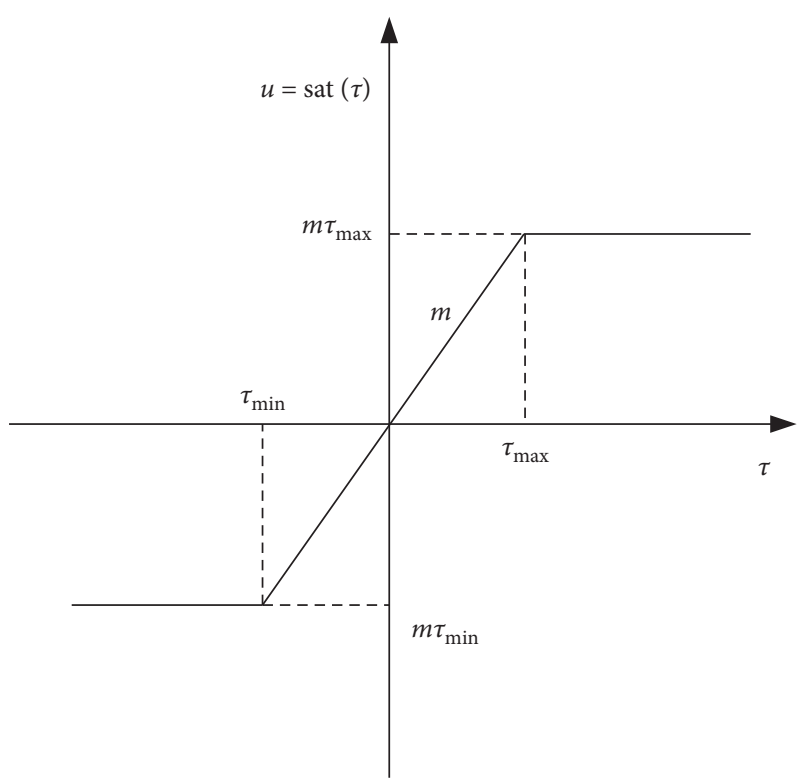

FIgURE 1: Saturation nonlinearity.

2.1. Saturation Nonlinearity. As shown in Figure 1, an unknown saturation in the control system can be described by

$$
u=\operatorname{sat}(\tau)= \begin{cases}m \tau_{\max }, & \tau(k) \geq \tau_{\max }, \\ m \tau(k), & \tau_{\min }<\tau(k)<\tau_{\max }, \\ m \tau_{\min }, & \tau(k) \leq \tau_{\min },\end{cases}
$$

where $u(k)$ and $\tau(k)$ represent the input and output signals, respectively, $\tau_{\min }$ and $\tau_{\max }$ are the minimum and maximum value of $\tau$, and $m$ is the slope of the linear region.

Saturation nonlinearity (1) can be represented in the form of

$$
u(k)=m \tau(k)+\varphi(k),
$$

with $\varphi(k)$ being a time variable disturbance signal.

It is obtained from (1) that

$$
\varphi(k)= \begin{cases}m\left(\tau_{\max }-\tau(k)\right), & \tau(k) \geq \tau_{\max }, \\ 0, & \tau_{\min }<\tau(k)<\tau_{\max }, \\ m\left(\tau_{\min }-\tau(k)\right), & \tau(k) \leq \tau_{\min },\end{cases}
$$

where $\varphi(k)$ is defined as $\varphi(k)=u(k)-m \tau(k)$.

Remark 1. It is concluded from (3) that if the control effort $|\tau|$ exceeds $\tau_{\max }$, a disturbance signal will be produced to prevent $\tau$ from being further increasing. In this case, the output tracking control may be failed since no sufficient control effort is imposed to the plant. This case occurs when the tracking error is large. As a result, the key issue is to make the control effort to avoid increasing before saturation. To realize this object, an unknown nonlinear function should be effectively approximated and compensated.

In this paper, FLS will be utilized to approximate the unknown uncertain function in a nonlinear model. The considered control system is a strict-feedback nonlinear plant with unknown input saturation, which is shown in

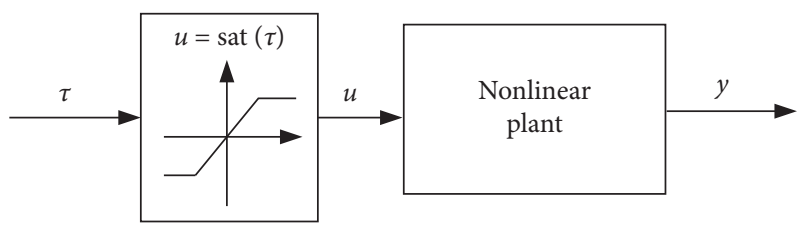

FIGURE 2: Saturation nonlinearity.

Figure 2. The nonlinear plant in Figure 2 will be analyzed in detail in the following section.

2.2. Nonlinear Plant in Triangular Form. For nonlinear plant, as shown in Figure 2, we consider a triangular nonlinear model in the form of

$$
\left\{\begin{array}{l}
x_{1}(k+1)=f_{1}\left(x_{1}(k)\right)+g_{1}\left(x_{1}(k)\right) x_{2}(k), \\
x_{i}(k+1)=f_{i}\left(\bar{x}_{i}(k)\right)+g_{i}\left(\bar{x}_{i}(k)\right) x_{i+1}(k), \\
x_{n}(k+1)=f_{n}(\bar{x}(k))+g_{n}(\bar{x}(k)) u(k), \\
y(k)=x_{1}(k),
\end{array}\right.
$$

where $\bar{x}(k)=\left[x_{1}(k), \ldots, x_{n}(k)\right]^{T}$ is a vector of system states, $\bar{x}_{i}(k)=\left[x_{1}(k), \ldots, x_{i}(k)\right]^{T}$ are vectors of system states with $i=2, \ldots, n-1$, and $f_{i}$ and $g_{i}, i=1, \ldots, n$, are smooth nonlinear functions.

For nonlinear system (4) preceded by input saturation (1) and a given bounded desired trajectory $x_{r}(k)$ with bounded derivatives up to $(n+1)$ th order, the control objective of this paper is to design an adaptive recursive dynamic surface control signal $\tau(k)$ such that the tracking error $e(k)$ is as small as possible. In particular, the input control signal is antiwindup, and the whole closed-loop system is Lyapunov stable.

Lemma 1 (see [26]). A class of nonlinear systems in the purefeedback form is

$$
\left\{\begin{array}{l}
x_{1}(k+1)=f_{1}\left(x_{1}(k)\right)+g_{1}\left(x_{1}(k)\right) x_{2}(k), \\
x_{i}(k+1)=f_{i}\left(\bar{x}_{i}(k)\right)+g_{i}\left(\bar{x}_{i}(k)\right) x_{i+1}(k), \\
x_{n}(k+1)=f_{n}\left(\bar{x}_{n}(k)\right)+g_{n}\left(\bar{x}_{n}(k)\right) u(k), \\
y(k)=x_{1}(k) .
\end{array}\right.
$$

Define alternative state variables $\bar{z}=T(\bar{x}(k))$ as

$$
\left\{\begin{array}{l}
z_{1}(k)=x_{1}(k), \\
z_{2}(k)=z_{1}(k+1), \\
z_{i+1}(k)=z_{i}(k+1), \quad i=2, \ldots, n-1, \\
y(k)=z_{1}(k)=x_{1}(k) .
\end{array}\right.
$$

System (5) can be represented as

$$
\left\{\begin{array}{l}
z_{1}(k+1)=z_{2}(k), \\
z_{i}(k+1)=z_{i+1}(k), \quad i=1, \ldots, n-1, \\
z_{n}(k+1)=\gamma_{n}\left(\bar{x}_{n}(k)\right)+\beta_{n}\left(\bar{x}_{n}(k)\right) u(k), \\
y(k)=x_{1}(k) .
\end{array}\right.
$$




$$
\begin{aligned}
\gamma_{n}\left(\bar{x}_{n}(k)\right) & =\sum_{j=1}^{n-1} \frac{\partial \gamma_{n-1}\left(\bar{x}_{n-1}(k)\right)}{\partial x_{j}(k)}\left[f_{j}\left(\bar{x}_{j}(k)\right)+g_{j}\left(\bar{x}_{j}(k)\right) x_{j+1}(k)\right]+\sum_{j=1}^{n-1} \frac{\partial \beta_{n-1}\left(\bar{x}_{n-1}(k)\right)}{\partial x_{j}(k)}\left[x_{i}(k)+\beta_{n-1}\left(\bar{x}_{n-1}(k)\right) f_{n}\left(\bar{x}_{n}(k)\right)\right] \\
\beta_{n}\left(\bar{x}_{n}(k)\right) & =\beta_{n-1}\left(\bar{x}_{n-1}(k)\right) g_{n}\left(\bar{x}_{n}(k)\right) \\
& =\prod_{i=1}^{n} g_{i}\left(\bar{x}_{i}(k)\right) .
\end{aligned}
$$

Furthermore, considering disturbance signal $\varphi(k),(7)$ can be reformed as

$$
\left\{\begin{array}{l}
z_{1}(k+1)=z_{2}(k) \\
z_{i}(k+1)=z_{i+1}(k), \quad i=1, \ldots, n-1, \\
z_{n}(k+1)=a_{n}\left(\bar{x}_{n}(k)\right)+b_{n}\left(\bar{x}_{n}(k)\right) \tau(k), \\
y(k)=x_{1}(k)=z_{1}(k)
\end{array}\right.
$$

where $a_{n}\left(\bar{x}_{n}(k)\right)$ and $b_{n}\left(\bar{x}_{n}(k)\right)$ are unknown functions defined as

$$
\begin{aligned}
a_{n}\left(\bar{x}_{n}(k)\right) & =\gamma_{n}\left(\bar{x}_{n}(k)\right)+\beta_{n}\left(\bar{x}_{n}(k)\right) \varphi(k) \\
& =\gamma_{n}\left(\bar{x}_{n}(k)\right)+d(k), \\
b_{n}\left(\bar{x}_{n}(k)\right) & =m \beta_{n}\left(\bar{x}_{n}(k)\right),
\end{aligned}
$$

with $d(k)$ being a disturbance signal produced when the control effort $|\tau|$ exceeds $\tau_{\text {max }}$.

Remark 2. It should be pointed out that, by coordinate transform $\bar{z}=T(\bar{x}(k))$ which is defined as in (6), there exists an inverse map $\bar{x}=T^{-1}(\bar{z}(k))$ representing the relationship of $\bar{x}$ and $\bar{z}(k)$. Thus, the obtained functions $a_{n}\left(\bar{x}_{n}(k)\right), b_{n}\left(\bar{x}_{n}(k)\right)$ are also unknown functions of $\bar{z}_{n}(k)$.

It is shown that, with the newly defined states (6) and the coordinate transform [26], the strict-feedback system (4) is now reformulated as a Brunovsky system (7) with an output signal $z_{1}(k)=x_{1}(k)$. Since $y(k)=z_{1}(k)=x_{1}(k)$ holds, the control objective (i.e., $y(k)$ tracks a reference signal $x_{d}(k)$ ) can be retained by controlling system (9). However, in the newly derived system (9), the states $z_{i}(k)$ are not directly measurable and the functions $a_{n}\left(\bar{x}_{n}(k)\right)$ and $b_{n}\left(\bar{x}_{n}(k)\right)$ are unknown. In this sense, the state-feedback control of the strict-feedback system (4) can be viewed as output feedback control of the canonical system (9). In the following, we will consider the output feedback control design of (9) to achieve control of (4).

2.3. Improved Observer. To estimate $z_{j}(k), j=2, \ldots, n$, a finite-time convergence observer is derived based on a highorder sliding mode (HOSM) observer (Levant's differentiator).

In the actual use of Levant's differentiator, it is found that when the function approaches stability, its small errors are amplified, and the rapid convergence rate will inevitably cause a chattering phenomenon. In short, when the error reaches the origin, it "does not stop," thus repeatedly crossing the origin and forming chattering, which has an impact on the actual system.

Theorem 1. The structure of the improved HOSM observer in this study is given as

$$
\left\{\begin{array}{l}
\widehat{z}_{1}(k+1)=v_{1}(k), \\
v_{1}(k)=\widehat{z}_{2}(k)-\lambda_{1} \eta_{1}(k), \\
\widehat{z}_{i}(k+1)=v_{i}(k), \\
v_{i}(k)=\widehat{z}_{i+1}(k)-\lambda_{i} \eta_{i}(k), \\
\widehat{z}_{n}(k+1)=v_{n}(k), \\
v_{n}(k)=\widehat{z}_{n+1}(k)-\lambda_{n} \eta_{n}(k), \\
\widehat{z}_{n+1}(k+1)=-\lambda_{n+1} \eta_{n+1}(k),
\end{array}\right.
$$

where the variable gain is defined as $\lambda_{i}=\left(\left(f a l\left(\eta_{i}(k), \delta, \kappa_{1}\right)\right) / \eta_{i}(k)\right), i=1,2, \ldots, n+1$ and nonlinear function $\mathrm{fal}\left(\eta_{i}(k), \delta, \kappa\right)$ is defined as

$$
\operatorname{fal}\left(\eta_{i}(k), \delta, \kappa_{i}\right)= \begin{cases}\left|\eta_{i}(k)\right|^{\kappa_{i}} \operatorname{sign}\left(\eta_{i}(k)\right), & \left|\eta_{i}(k)\right|>\delta, \\ \frac{\eta_{i}(k)}{\delta^{1-\kappa_{i}}}, & \left|\eta_{i}(k)\right| \leq \delta,\end{cases}
$$

with $\kappa_{i}=((n+1-i) /(n+2-i))$ and $\kappa$ and $\delta$ are adjustable design parameters. The value of $\kappa$ determines the degree of nonlinearity of the function and $\delta$ is the width of the linear interval, as shown in Figure 3:

$$
\left\{\begin{array}{l}
\eta_{1}(k)=\widehat{z}_{1}(k)-x_{1}(k)=\widehat{z}_{1}(k)-z_{1}(k), \\
\eta_{j}(k)=\widehat{z}_{j}(k)-v_{j-1}(k), \quad j=2, \ldots, n+1,
\end{array}\right.
$$

satisfying

$$
\left\{\begin{array}{l}
\left|\tilde{z}_{i}(k)\right| \leq \mu_{i} \varepsilon^{((n-i+1) /(n+1))}, \\
\left|\eta_{i}(k)\right| \leq \varsigma_{i} \varepsilon^{((n-i) /(n+1))}, \quad i=1, \ldots, n+1,
\end{array}\right.
$$

where $\widetilde{z}_{i}(k)=\widehat{z}_{i}(k)-z_{i}(k)(i=1, \ldots, n)$ represent the $o b$ server errors and $\mu_{i}$ and $\varsigma_{i}$ are some positive constants with $\left|\widetilde{z}_{1}(k)\right| \leq \varepsilon$.

\section{It can be further obtained that}

$$
\left\|\tilde{z}_{i}(k)\right\| \leq \mu=\max \left\{\mu_{i} \varepsilon^{((n-i+1) /(n+1))}\right\} \quad i=1, \ldots, n .
$$

It should be pointed out that an improved HOSM observer (11) can achieve finite-time error convergence. This 


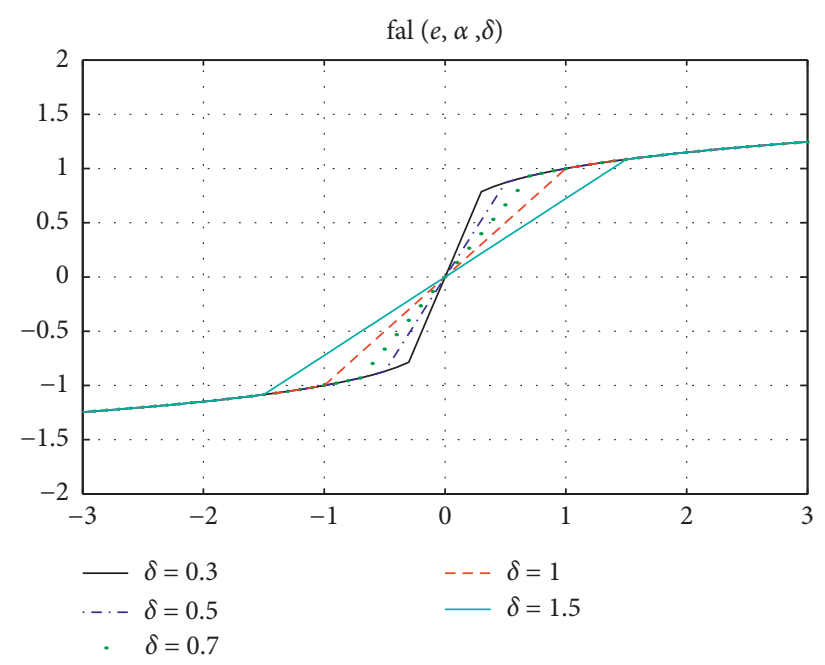

(a)

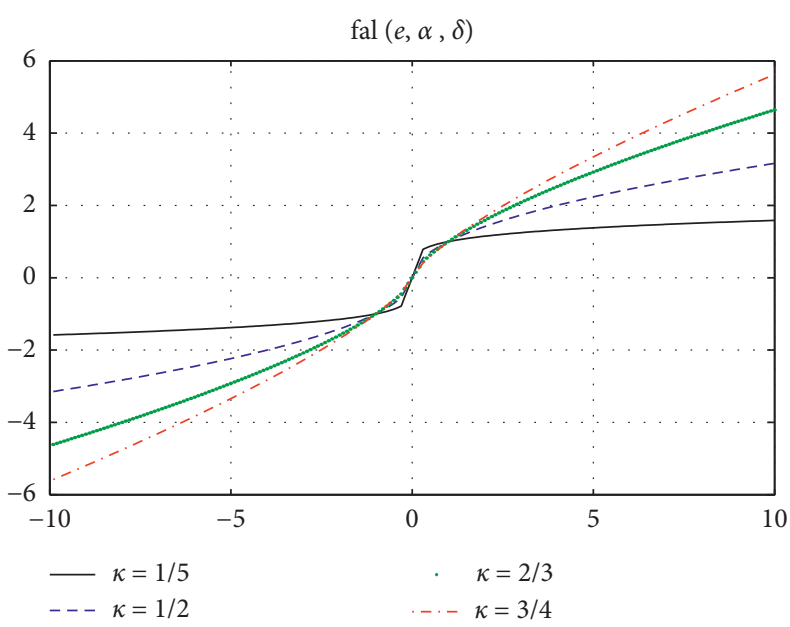

(b)

FIgURE 3: Output signal of the nonlinear function fal $(\cdot)$. (a) Output of fal(.) with different $\delta$. (b) Output of fal $(\cdot)$ with different $\kappa$.

means that a controller and an observer can be designed separately so that the combined controller-observer output feedback preserves the main features of the controller with a full state available.

The nonlinear function fal (.) has the design concept of "small error amplification, large error reduction" and retains the advantage of fast convergence of high-order sliding mode in terms of convergence rate. Different from the nonlinear term of Levant's differentiator, $\left(\left(\eta_{i}(k)\right) / \delta^{1-\beta}\right)<\left|\eta_{i}(k)\right|^{\beta} \operatorname{sign}\left(\eta_{i}(k)\right)$ exists for any $\delta \in(0,1)$ and $\kappa \in(0,1)$ when $|e| \in(0, \delta)$, which can effectively deal with the stable chattering problem of the system.

Proof.

$$
\left\{\begin{array}{l}
\widehat{z}_{1}(k+1)=v_{1}(k), \\
v_{1}(k)=\widehat{z}_{2}(k)-\lambda_{1} \eta_{1}(k), \\
\widehat{z}_{i}(k+1)=v_{i}(k), \\
v_{i}(k)=\widehat{z}_{i+1}(k)-\lambda_{i} \eta_{i}(k), \\
\widehat{z}_{n}(k+1)=v_{n}(k), \\
v_{n}(k)=\widehat{z}_{n+1}(k)-\lambda_{n} \eta_{n}(k), \\
\widehat{z}_{n+1}(k+1)=-\lambda_{n+1} \eta_{n+1}(k) .
\end{array}\right.
$$

The observer (16) can be represented as

$$
\begin{aligned}
& \left\{\begin{array}{l}
\widehat{z}_{1}(k+1)=\widehat{z}_{2}(k)-\lambda_{1} \eta_{1}(k), \\
\widehat{z}_{2}(k+1)=\widehat{z}_{3}(k)-\lambda_{2} \eta_{2}(k), \\
\vdots \\
\widehat{z}_{i}(k+1)=\widehat{z}_{i+1}(k)-\lambda_{i} \eta_{i}(k), \\
\vdots \\
\widehat{z}_{n}(k+1)=\widehat{z}_{n+1}(k)-\lambda_{n} \eta_{n}(k), \\
\widehat{z}_{n+1}(k+1)=-\lambda_{n+1} \eta_{n+1}(k),
\end{array}\right. \\
& v_{i}(k)=\widehat{z}_{i+1}(k)-\lambda_{i} \eta_{i}(k), \quad i=1, \ldots,(n+1) .
\end{aligned}
$$

The specific expressions $\lambda_{i}$ on both sides of the error boundary are different, so they are discussed separately here. When $\left|\eta_{i}\right| \leq \delta, \lambda_{i}=\left(1 /\left(\delta^{1-\kappa_{i}}\right)\right), i=1, \ldots,(n+1)$, the observer is linear feedback. Its linear feedback form is as follows:

$$
\begin{aligned}
& \left\{\begin{array}{l}
\widehat{z}_{1}(k+1)=\widehat{z}_{2}(k)-\lambda_{1} \widetilde{z}_{1}(k), \\
\widehat{z}_{2}(k+1)=\widehat{z}_{3}(k)-\lambda_{2} \widetilde{z}_{2}(k), \\
\vdots \\
\widehat{z}_{i}(k+1)=\widehat{z}_{i+1}(k)-\lambda_{i} \widetilde{z}_{i}(k), \\
\vdots \\
\widehat{z}_{n}(k+1)=\widehat{z}_{n+1}(k)-\lambda_{n} \widetilde{z}_{n}(k), \\
\widehat{z}_{n+1}(k+1)=-\lambda_{n+1} \widetilde{z}_{n+1}(k),
\end{array}\right. \\
& v_{i}(k)=\widehat{z}_{i+1}(k)-\lambda_{i} \widetilde{z}_{i}(k), \quad i=1, \ldots,(n+1) \text {. }
\end{aligned}
$$


Define the observation error $\widetilde{z}_{i}(k)=\widehat{z}_{i}(k)-z_{i}(k)(i=$ $1, \ldots, n+1)$ and the time derivative of $\widetilde{z}_{i}(k)$ is

$$
\left\{\begin{array}{l}
\widetilde{z}_{1}(k+1)=\widetilde{z}_{2}(k)-\lambda_{1}\left(\widehat{z}_{1}(k)-z_{1}(k)\right), \\
\widetilde{z}_{2}(k+1)=\widetilde{z}_{3}(k)-\lambda_{2}\left(\widehat{z}_{2}(k)-z_{2}(k)\right), \\
\vdots \\
\widetilde{z}_{i}(k+1)=\widetilde{z}_{i+1}(k)-\lambda_{i}\left(\widehat{z}_{i}(k)-z_{i}(k)\right), \\
\vdots \\
\widetilde{z}_{n}(k+1)=\widetilde{z}_{n+1}(k)-\lambda_{n}\left(\widehat{z}_{n}(k)-z_{n}(k)\right), \\
\widetilde{z}_{n+1}(k+1)=-\lambda_{n+1}\left(\widehat{z}_{n+1}(k)-z_{n+1}(k)\right) .
\end{array}\right.
$$

Defining $\bar{z}_{i}(k)=\left[\widetilde{z}_{1}(k), \ldots, \widetilde{z}_{n+1}(k)\right]$, the appropriate parameter $\lambda_{i}$ is selected and

$$
A_{0}=\left[\begin{array}{ccccc}
-\lambda_{1} & 1 & 0 & \ldots & 0 \\
-\lambda_{2} & 0 & 1 & \ldots & 0 \\
\vdots & \vdots & \vdots & \ddots & \vdots \\
-\lambda_{n} & 0 & 0 & \ldots & 1 \\
-\lambda_{n+1} & 0 & 0 & \ldots & 0
\end{array}\right]
$$

is a strict Hurwitz matrix. follows:

The selected Lyapunov candidate function is defined as

$$
V_{0}(k)=\bar{z}_{i}^{T}(k) P_{i} \bar{z}_{i}(k)
$$

The derivative of the candidate function is selected as

$$
\begin{aligned}
V_{0}(k+1) & =\bar{z}_{i}^{T}(k+1) P_{i} \bar{z}_{i}(k)+\bar{z}_{i}^{T}(k) P_{i} \bar{z}_{i}(k+1) \\
& =\bar{z}_{i}^{T}(k) A_{0}^{T} P_{i} \bar{z}_{i}(k)+\bar{z}_{i}^{T}(k) P_{i} A_{0} \bar{z}_{i}(k) \\
& =\bar{z}_{i}^{T}\left(A_{0}^{T} P_{i} \bar{z}_{i}(k)+\bar{z}_{i}^{T}(k) P_{i} A_{0}\right) \bar{z}_{i}(k) .
\end{aligned}
$$

Giving $Q_{i}^{T}=Q_{i}>0$. Then, there exists a positive definite matrix $P_{i}^{T}=P_{i}>0$ and

$$
A_{0}^{T} P_{i}+P_{i} A_{0}=-Q_{i} .
$$

Therefore, it is obtained that

$$
V_{0}(k+1) \leq-q_{\min }\left(Q_{i}\right)\left\|\bar{z}_{i}(k)\right\|,
$$

where $q_{\min }\left(Q_{i}\right)$ is a minimum eigenvector in matrix $Q_{i}$.

When $\quad\left|\eta_{i}\right|>\delta, \quad \lambda_{i}=\left|\eta_{i}(k)\right|^{\kappa_{i}} \operatorname{sign}\left(\eta_{i}(k)\right), i=1, \ldots$, $(n+1)$, the observer is

$$
\left\{\begin{array}{l}
\widehat{z}_{1}(k+1)=\widehat{z}_{2}(k)-\left|\widetilde{z}_{1}(k)\right|^{\kappa_{1}} \operatorname{sign}\left(\eta_{1}(k)\right) \widetilde{z}_{1}(k), \\
\widehat{z}_{2}(k+1)=\widehat{z}_{3}(k)-\left|\widetilde{z}_{2}(k)\right|^{\kappa_{2}} \operatorname{sign}\left(\eta_{2}(k)\right) \widetilde{z}_{2}(k), \\
\vdots \\
\widehat{z}_{i}(k+1)=\widehat{z}_{i+1}(k)-\left|\widetilde{z}_{i}(k)\right|^{\kappa_{i}} \operatorname{sign}\left(\eta_{i}(k)\right) \widetilde{z}_{i}(k), \\
\vdots \\
\widehat{z}_{n}(k+1)=\widehat{z}_{n+1}(k)-\left|\widetilde{z}_{n}(k)\right|^{\kappa_{n}} \operatorname{sign}\left(\eta_{n}(k)\right) \widetilde{z}_{n}(k), \\
\widehat{z}_{n+1}(k+1)=-\left|\widetilde{z}_{n+1}(k)\right|^{\kappa_{n+1}} \operatorname{sign}\left(\eta_{n+1}(k)\right) \widetilde{z}_{n+1}(k) .
\end{array}\right.
$$

Define the observation error $\widetilde{z}_{i}(k)=\widehat{z}_{i}(k)-z_{i}(k)(i=$ $1, \ldots, n+1)$ and select appropriate parameters $\kappa_{i}$.
According to the literature [27], the observation error can be stabilized in a finite time.

It is not unusual that a sliding mode is finally attained in finite time. This finite-time property of sliding mode observers is very attractive.

Remark 3. For the nonlinear system in the form of (4), lots of adaptive fuzzy control schemes have been developed based on state feedback in the past decades. In this case, one assumption is that all the system states are available when implementing these algorithms. Usually, not all the states are available in practice. Accordingly, a state observer is needed if not all states are available for feedback control. In the following, we design a recursive fuzzy DSC signal based on transform (9), where the states are obtained by HOSM observer (11).

\section{Recursive Dynamic Surface Controller Design}

Assumption 1. It is assumed that $f_{i}\left(x_{i}(k)\right), i=1, \ldots, n$, are totally unknown smooth functions while $g_{i}\left(x_{i}(k)\right)$ are known positive or negative functions.

Remark 4. In control systems, the assumption on $g_{i}(x(k))$ is practical since $g_{i}(x(k))$ is very important in control signal design. In most of the published results, assumptions should be made before controller design to make the control design possible. If $g_{i}(x)$ are totally unknown, control may be failed since $g_{i}(x(k))$ is a time variable or even 0 , significantly affecting control performance. In the worst case, instability may be occurred.

In the control design, the following dynamic sliding surfaces will be used:

$$
\left\{\begin{array}{l}
e_{1}(k)=\widehat{z}_{1}(k)-x_{r}(k), \\
e_{2}(k)=e_{1}(k+1)+x_{r}(k+1)-\vartheta_{1}(k), \\
e_{i}(k)=e_{k-1}(k+1)+\vartheta_{i-2}(k+1)-\vartheta_{k-1}(k), \\
e_{n}(k)=e_{n-1}(k+1)+\vartheta_{n-2}(k+1)-\vartheta_{n-1}(k),
\end{array}\right.
$$

where $i=2, \ldots, n-1$ and $\vartheta(k)$ is the virtual control signal.

Based on the above-defined dynamic sliding surfaces, an adaptive recursive command filtered output feedback control scheme will be developed in detail with fuzzy compensation.

The process is carried out based on the coming steps:

Step 1: from (26), the state tracking error is defined as

$$
e_{1}(k)=\widehat{z}_{1}(k)-x_{r}(k),
$$

where $x_{r}(k)$ is the reference signal.

Choose the Lyapunov function candidate as

$$
V_{1}=\frac{1}{2} e_{1}^{2}(k) \text {. }
$$

Then, the first-order difference of (28) is computed as 


$$
\begin{aligned}
\Delta V_{1} & =\frac{1}{2} e_{1}^{2}(k+1)-\frac{1}{2} e_{1}^{2}(k) \\
& =\frac{1}{2}\left[\widehat{z}_{1}(k+1)-x_{r}(k+1)\right]^{2}-\frac{1}{2} e_{1}^{2}(k) .
\end{aligned}
$$

According to the DSC method, a virtual control signal is defined as $\vartheta_{1}(k)=x_{r}(k+1)$. It is very important that, in order to obtain $x_{r}(k+1)$, let $x_{r}(k)$ pass through a tracking differentiator (TD) in the form of

$$
\left\{\begin{array}{l}
\chi_{1}(k+1)=\chi_{2}(k), \\
\chi_{2}(k+1)=f s t\left(\chi_{1}(k)-x_{r}(k), \chi_{2}(k), r, h_{0}\right),
\end{array}\right.
$$

where $\chi_{1}(k)$ and $\chi_{2}(k)$ are the states of TD, $h_{0}$ is a filter coefficient, and $r$ is a velocity factor. According to [28], the nonlinear function fst $(\cdot)$ is defined as

$$
\text { fst }(\cdot)= \begin{cases}-a r, & |a| \leq d, \\ -r \operatorname{sign}(a), & |a|>d,\end{cases}
$$

with

$$
\begin{aligned}
a & = \begin{cases}\chi_{2}+\frac{c}{h_{0}}, & |c|<d_{0}, \\
\chi_{2}+\operatorname{sign}(c) \frac{\left(a_{0}-d\right)}{2}, & |c|>d_{0},\end{cases} \\
d & =r h_{0}, \\
d_{0} & =d h_{0}, \\
c & =\chi_{1}-x_{r}+h_{0} \chi_{2}, \\
a_{0} & =\sqrt{d^{2}+8 r|c|},
\end{aligned}
$$

with $h_{0}$ being the sample period.

Then, the second error surface is

$$
e_{2}=\widehat{z}_{2}-\vartheta_{1}
$$

with $\vartheta_{1}(k)$ being the virtual control signal in Step 1 . Substituting (33) into (29) yields

$$
\begin{aligned}
\Delta V_{1} & =\frac{1}{2}\left[\widehat{z}_{2}(k)-x_{r}(k+1)\right]^{2}-\frac{1}{2} e_{1}^{2}(k) \\
& =\frac{1}{2}\left[e_{2}(k)+\vartheta_{1}-x_{r}(k+1)\right]^{2}-\frac{1}{2} e_{1}^{2}(k) \\
& =\frac{1}{2} e_{2}^{2}(k)-\frac{1}{2} e_{1}^{2}(k) .
\end{aligned}
$$

Step i $(2 \leq i<n)$ : the $i$ th error surface is defined as

$$
e_{i}(k)=\widehat{z}_{i}(k)-\vartheta_{i-1}(k),
$$

where $\vartheta_{i-1}(k)$ represents the intermediate virtual control signal in the last step. The virtual control signal in this step is

$$
\vartheta_{i}(k)=\vartheta_{i-1}(k+1),
$$

where $\vartheta_{i-1}(k+1)$ is the dynamic of the virtual control signal and is very important in the DSC design spirit. In this design, similar to Step 1, let $\vartheta_{i-1}(k)$ pass through a TD to obtain $\vartheta_{i-1}(k+1)$ as

$$
\left\{\begin{array}{l}
\chi_{i 1}(k+1)=\chi_{i 2}(k), \\
\chi_{i 2}(k+1)=f s t\left(\chi_{i 1}(k)-\vartheta_{i-1}(k), \vartheta_{i-1}(k), r, h_{0}\right),
\end{array}\right.
$$

with $\chi_{i 2}(k)$ being tracking signal $\vartheta_{i-1}(k+1)$.

Choose the Lyapunov function candidate as

$$
V_{i}=V_{i-1}+\frac{1}{2} e_{i}^{2}(k) .
$$

Then, the first-order difference of (38) is computed as

$$
\begin{aligned}
\Delta V_{i} & =\frac{1}{2}\left[\widehat{z}_{i+1}(k)-\vartheta_{i-1}(k+1)\right]^{2}-\frac{1}{2} e_{i}^{2}(k)+\Delta V_{i-1} \\
& =\frac{1}{2} e_{i+1}^{2}(k)-\frac{1}{2} e_{i}^{2}(k)+\Delta V_{i-1} \\
& =\frac{1}{2} e_{i+1}^{2}(k)-\frac{1}{2} e_{1}^{2}(k) .
\end{aligned}
$$

Step $n$ : define the last error surface as

$$
e_{n}(k)=\widehat{z}_{n}(k)-\vartheta_{n-1}(k),
$$

with $\vartheta_{n-1}$ being the last intermediate virtual control signal.

Choose the Lyapunov function candidate as

$$
V_{n}=V_{n-1}+\frac{1}{2} e_{n}^{2}(k) .
$$

Then, the first-order difference of (41) is computed as

$$
\begin{aligned}
\Delta V_{n} & =\frac{1}{2} e_{n}^{2}(k+1)-\frac{1}{2} e_{n}^{2}(k)+\Delta V_{n-1} \\
& =\frac{1}{2}\left[\widehat{z}_{n}(k+1)-\vartheta_{n-1}(k+1)\right]^{2}-\frac{1}{2} e_{n}^{2}(k)+\Delta V_{n-1} \\
& =\frac{1}{2}\left[a_{n}\left(\widehat{z}_{n}(k)\right)+b_{n}\left(\widehat{z}_{n}(k)\right) \tau(k)-\vartheta_{n-1}(k+1)\right]^{2}-\frac{1}{2} e_{n}^{2}(k)+\Delta V_{n-1} .
\end{aligned}
$$


Lemma 2. Let $f(x)$ be a continuous function defined on a compact set $\Omega$. Then, for any scalar $\varepsilon>0$, there exists an FLS $\theta^{T} \sigma(x)$ such that

$$
\sup _{x \in \Omega}\left|f(x)-\theta^{T} \sigma(x)\right| \leq \varepsilon,
$$

where $\theta=\left[\theta_{1}, \ldots, \theta_{N}\right]^{T}$ is the ideal constant weight vector and $\sigma(x)=\left[p_{1}(x), p_{2}(x), \ldots, p_{N}(x)\right]^{T} \sum_{i=1}^{N} p_{i}(x)$ is the basis function vector, with $N>1$ being the number of the fuzzy rules and $p_{i}$ being chosen as Gaussian functions, that is, for $i=1,2, \ldots, N, p_{i}(x)=\exp \left[\left(\left(-\left(x-\mu_{i}\right)^{T}\left(x-\mu_{i}\right)\right)\left(\eta_{i}^{2}\right)\right)\right]$, where $\mu_{i}=\left[\mu_{i 1}, \mu_{i 2}, \ldots, \mu_{i n}\right]^{T}$ is the center vector and $\eta_{i}$ is the width of the Gaussian function.

It is known that, from the approximation property of FLS, there exists a FLS satisfying the following equation:

$$
F(k)=\frac{-a_{n}\left(z_{n}(k)\right)+\vartheta_{n-1}(k+1)}{b_{n}\left(z_{n}(k)\right)}=\theta^{T} \xi\left(z_{n}(k)\right)+\varepsilon .
$$

Choose the actual control signal and adaptive law to be

$$
\tau(k)=\widehat{W}(k)\left\|\xi\left(\widehat{z}_{n}(k)\right)\right\|,
$$

$$
\widehat{W}(k+1)=\gamma\left[\left\|\xi\left(\widehat{z}_{n}(k)\right)\right\| e_{n}(k+1)-\sigma \widehat{W}(k)\right]+\widehat{W}(k),
$$

with $W(k)=\left\|\theta^{T}\right\|, \widetilde{W}(k)=\widehat{W}(k)-W^{*} \cdot \gamma, \sigma$ are adjustable parameters.

Differentiating $e_{n}(k)$ yields

$$
\begin{aligned}
e_{n}(k+1) & =a_{n}\left(\widehat{z}_{n}(k)\right)+b_{n}(\widehat{z}(k)) \tau(k)-\vartheta_{n-1}(k+1) \\
& =\widetilde{W}(k)\left\|\xi\left(\widehat{z}_{n}(k)\right)\right\|+\varepsilon .
\end{aligned}
$$

Using $\|\xi(\cdot)\|^{2} \leq 1$ and according to Young's inequality, $e_{n}^{2}(k+1)$ can be written as

$$
\begin{aligned}
e_{n}^{2}(k+1) & =\left[\tilde{W}(k)\left\|\xi\left(\widehat{z}_{n}(k)\right)\right\|+\varepsilon\right]^{2} \\
& \leq \tilde{W}^{2}(k)\left\|\xi\left(\widehat{z}_{n}(k)\right)\right\|^{2}+\varepsilon^{2} \\
& \leq \tilde{W}^{2}(k)+\varepsilon^{2} .
\end{aligned}
$$

Substituting (48) into (42) yields

$$
\begin{aligned}
\Delta V_{n} & \leq \frac{1}{2}\left(\tilde{W}^{2}(k)+\varepsilon^{2}\right)-\frac{1}{2} e_{n}^{2}(k)+\Delta V_{n-1} \\
& =\frac{1}{2}\left(\tilde{W}^{2}(k)+\varepsilon^{2}\right)-\frac{1}{2} e_{1}^{2}(k) .
\end{aligned}
$$

Proof. Consider a Lyapunov candidate function in the form of

$$
V(k)=\sum_{i=1}^{n} \frac{1}{2} e_{i}^{2}(k)+\frac{1}{\gamma} \widetilde{W}^{2}(k)
$$

where $\gamma$ is a positive design parameter.

Differentiating $V(k)$ yields

$$
\Delta V(k)=\Delta V_{n}(k)+\frac{1}{\gamma}\left[\tilde{W}^{2}(k+1)-W^{2}(k)\right] .
$$

Using (46) obtains

$$
\begin{aligned}
\widetilde{W}(k+1) & =\widehat{W}(k+1)-W^{*}, \\
& =\widehat{W}(k)+\gamma\left[\left\|\xi\left(\widehat{z}_{n}(k)\right)\right\| e_{n}(k+1)-\sigma \widehat{W}(k)\right]-W^{*} \\
& =(1-\gamma \sigma) \widehat{W}(k)+\gamma\left\|\xi\left(\widehat{z}_{n}(k)\right)\right\| e_{n}(k+1)-W^{*} \\
& =(1-\gamma \sigma) \widetilde{W}(k)-\gamma \sigma W^{*}+\gamma\left\|\xi\left(\widehat{z}_{n}(k)\right)\right\| e_{n}(k+1) .
\end{aligned}
$$

Next,

$$
\begin{aligned}
\tilde{W}^{2}(k+1)-\tilde{W}^{2}(k)= & {\left[(1-\gamma \sigma) \tilde{W}(k)-\gamma \sigma W^{*}+\gamma\left\|\xi\left(\widehat{z}_{n}(k)\right)\right\| e_{n}(k+1)\right]^{2}-\widetilde{W}^{2}(k) } \\
= & (1-\gamma \sigma)^{2} \tilde{W}^{2}(k)+\left(\gamma \sigma W^{*}\right)^{2}+\gamma^{2}\left\|\xi\left(\widehat{z}_{n}(k)\right)\right\|^{2} e_{n}^{2}(k+1)-2(1-\gamma \sigma) \gamma \sigma W^{*} \tilde{W}(k) \\
& +2(1-\gamma \sigma) \gamma\left\|\xi\left(\widehat{z}_{n}(k)\right)\right\| e_{n}(k+1) \tilde{W}(k)-2 \gamma^{2} \sigma W^{*}\left\|\xi\left(\widehat{z}_{n}(k)\right)\right\| e_{n}(k+1)-\tilde{W}^{2}(k) .
\end{aligned}
$$

Using $\|\xi(\cdot)\|^{2} \leq 1$ and according to Young's inequality can yield

$$
\begin{gathered}
2(1-\gamma \sigma) \gamma \sigma W^{*} \tilde{W}(k) \leq \gamma \sigma(1-\gamma \sigma)\left(W^{* 2}+\tilde{W}^{2}(k)\right), \\
2 \gamma^{2} \sigma W^{*}\left\|\xi\left(\widehat{z}_{n}(k)\right)\right\| e_{n}(k+1) \leq \gamma^{2} \sigma\left(e_{n}^{2}(k+1)+W^{* 2}\right), \\
2(1-\gamma \sigma) \gamma\left\|\xi\left(\widehat{z}_{n}(k)\right)\right\| e_{n}(k+1) \tilde{W}(k) \leq \gamma(1-\gamma \sigma)\left(e_{n}^{2}(k+1)+\widetilde{W}^{2}(k)\right) .
\end{gathered}
$$


TABle 1: Value of coefficients in Example 1.

\begin{tabular}{lcccccc}
\hline Parameter & $k_{1}$ & $k_{2}$ & $r_{1}$ & $r_{2}$ & $\beta_{1}$ \\
\hline Value & 10 & 10 & 10 & 20 & 15 & 5 \\
Parameter & $\kappa_{1}$ & $\kappa_{2}$ & $\kappa_{3}$ & $h_{0}$ & $\Gamma$ & 0 \\
Value & 0.75 & $2 / 3$ & 0.5 & 0.01 & $1.5 * I_{25}$ & 0.01 \\
\hline
\end{tabular}

Substituting (54) into (53) yields

$$
\widetilde{W}^{2}(k+1)-\widetilde{W}^{2}(k) \leq a \widetilde{W}^{2}(k)+b W^{* 2}+c e_{n}^{2}(k+1),
$$

where

$$
\begin{aligned}
& a=2(\gamma \sigma)^{2}-3 \gamma \sigma+\gamma-\gamma^{2} \sigma-1, \\
& b=2(\gamma \sigma)^{2}-\gamma \sigma-\gamma^{2} \sigma, \\
& c=\gamma^{2}-2 \gamma^{2} \sigma+\gamma .
\end{aligned}
$$

Substituting (48) into (55) can obtain

$$
\widetilde{W}^{2}(k+1)-\widetilde{W}^{2}(k) \leq(a+c) \tilde{W}^{2}(k)+b W^{* 2}+c \varepsilon^{2} .
$$

Substituting (57) and (49) into (51) can obtain

$$
\begin{aligned}
\Delta V(k) & \leq-\frac{1}{2} e_{1}^{2}(k)+\frac{1}{\gamma}\left[\left(a+c+\frac{\gamma}{2}\right) \widetilde{W}^{2}(k)+b W^{* 2}+\left(c+\frac{\gamma}{2}\right) \varepsilon^{2}\right] \\
& \leq-\frac{1}{2} e_{1}^{2}(k)+\frac{1}{\gamma}\left(a+c+\frac{\gamma}{2}\right) \tilde{W}^{2}(k)+\mu,
\end{aligned}
$$

where $\mu=(1 / \gamma)\left[b W^{* 2}+(c+(\gamma / 2)) \varepsilon^{2}\right]$, selecting the appropriate parameters content $\gamma, \sigma$ content $a+c+(\gamma / 2)<0$. Once the error variables $\left|e_{1}(k)\right|>\sqrt{2 \mu}$ are valid, then $\Delta V(k) \leq 0$ is obtained.

This completes the proof.

Remark 5. We employ TD (31) to eliminate the problem of "explosion of complexity" in the traditional backstepping scheme. It is noted that, in the developed RDSC scheme, TD is superior to the first-order filter for its excellent filtering performance of the high-frequency noises. As a result, the proposed DSC scheme in this research increases control performance by reducing controller complexity and filtering high-frequency noises. In the following, we will illustrate this scheme by simulation on a strict-feedback nonlinear system and a "CSTRs" nonlinear system compared to the traditional SMC method.

\section{Simulation Study}

In this section, two numerical examples are simulated to validate the effectiveness of the fuzzy DSC algorithm proposed in the above sections.

Example 1. To further validate our proposed adaptive algorithm, consider the following strict-feedback nonlinear system:

$$
\left\{\begin{array}{l}
x_{1}(k+1)=f_{1}\left(x_{1}(k)\right)+g_{1}\left(x_{1}\right) x_{2}(k), \\
x_{2}(k+1)=f_{2}\left(\bar{x}_{2}(k)\right)+g_{2}\left(\bar{x}_{2}(k)\right) \tau(k),
\end{array}\right.
$$

with input saturation parameter chosen as $\tau_{\min }=-3, \tau_{\max }=$ $3, m=2$ and unknown nonlinear functions defined as

$$
\begin{aligned}
& f_{1}\left(x_{1}(k)\right)=x_{1}^{2}(k), \\
& g_{1}\left(x_{1}(k)\right)=1+0.1 \sin \left(x_{1}(k)\right), \\
& f_{2}\left(\bar{x}_{2}(k)\right)=0.2 e^{-x_{2}(k)}+x_{1}(k) \sin \left(x_{2}(k)\right), \\
& g_{2}\left(x_{2}(k)\right)=1 .
\end{aligned}
$$

The aim of this example is also to make the output signal $y=x_{1}$ to track a provided reference signal $x_{r}=\sin (t)$.

According to the analysis in Section 2, it is obtained by coordinate transform that

$$
\begin{aligned}
& \gamma_{2}\left(\bar{x}_{2}(k)\right)=\left(x_{1}(k)+0.1 x_{2}(k) \cos \left(x_{2}(k)\right)\right)\left(x_{2}+1+0.1 \sin \left(x_{1}(k)\right)\right) x_{2}(k)+\left(1+0.1 \sin x_{1}(k)\right)\left(0.2 e^{-x_{2}(k)}+x_{1}(k) \sin \left(x_{2}(k)\right)\right) \\
& \beta_{2}\left(\bar{x}_{2}(k)\right)=1+0.2 \sin \left(x_{1}(k)\right) .
\end{aligned}
$$

In this simulation, values of simulation coefficients are given as in Table 1.

For comparison, the control performance by traditional sliding mode control (SMC) technique is employed as the control signal, which is in the form of

$$
\tau(k)=-\left(\frac{1}{b_{n}}\left(\bar{x}_{n}(k)\right)\left(K s+\lambda_{1} e_{2}(k)-x_{r}(k+2)+\widehat{a}_{2}\left(\bar{x}_{2}(k)\right)+\omega\right)\right),
$$

where $\omega$ is a robust term defined as

$$
\omega=\varepsilon_{M} \operatorname{signs}\left(\bar{e}_{2}(k)\right)
$$

with

$$
s=\lambda_{1} e_{1}(k)+\lambda_{2} e_{2}(k)
$$

where $\lambda_{1}$ and $\lambda_{2}$ are positive constants. In simulation, the values of SMC parameters are chosen to be $K=5, \lambda_{1}=1, \lambda_{2}=1, \varepsilon_{M}=0.1$.

The simulation results are depicted in Figures 4-7. From the simulation results, the following advantages are obtained by using the proposed algorithm. First, a better control 


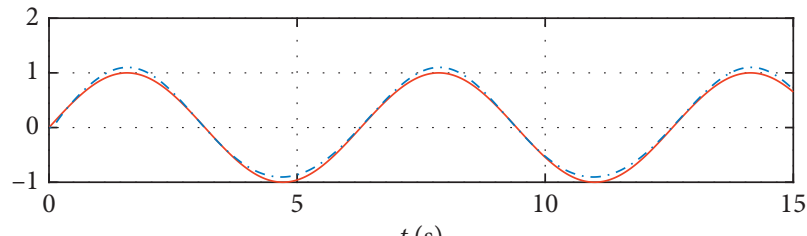

$t(\mathrm{~s})$

$$
\begin{array}{r}
x_{r} \\
--x_{1}
\end{array}
$$

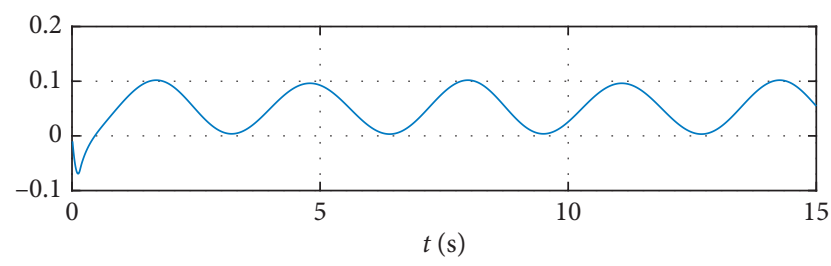

$-e_{1}$

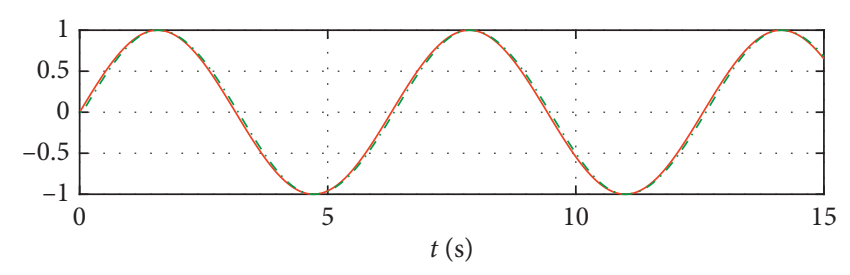

$-x_{r}$

$\cdot-\chi_{11}$

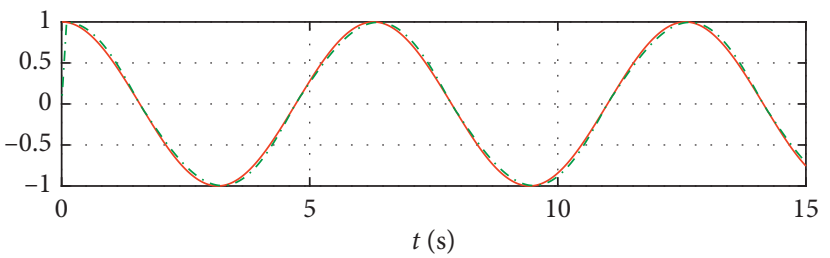

$-d x_{r}$

$\ldots-X_{12}$

(a)

(b)

FIgURE 4: Simulation results of Example 1. (a) DSC tracking performance in Example 1. (b) States of TD 1
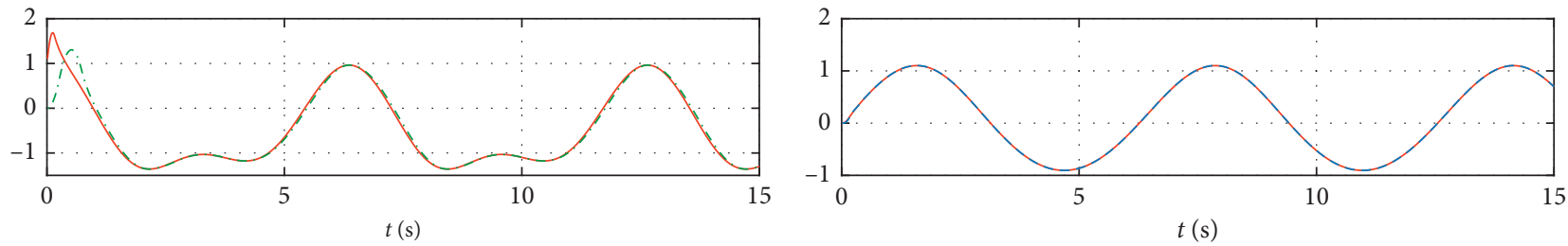

$$
\begin{aligned}
& \alpha_{1} \\
& --\chi_{21}
\end{aligned}
$$

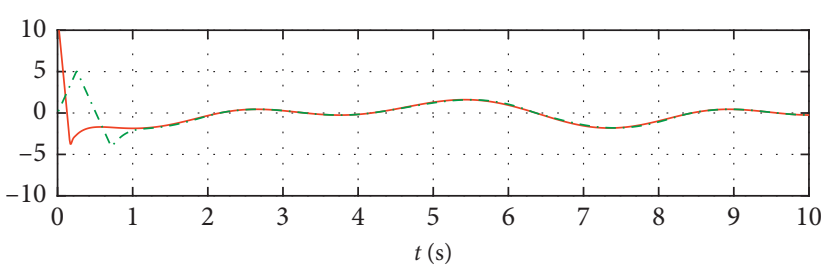

$-\dot{\alpha}_{1}$

$-\chi_{22}$

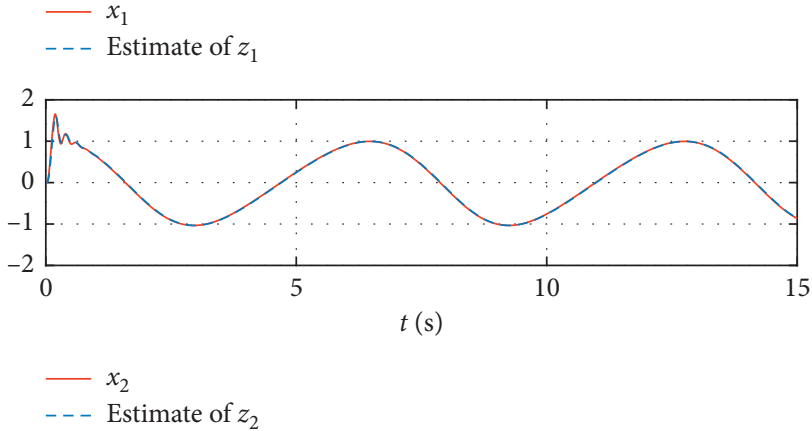

(b)

Figure 5: Simulation results of Example 1. (a) States of TD2. (b) States of ESO.

performance is achieved with a smaller tracking error, which can be seen by comparing the tracking curves in Figure 4(a) and Figure 6(a). Next, derivatives of reference signal $x_{r}$ and virtual control signal $\vartheta_{1}$ are both available without highfrequency noises, which are shown in Figure 4(b) and Figure 5(a). Moreover, an output feedback control is realized by applying HOSM with the state curves shown in Figure 5(b). In particular, there is no chattering phenomenon by the proposed algorithm which can be seen by comparing the curves in Figure 4(a) and Figure 6(a).
Example 2. To validate the proposed DSC scheme, consider a "continuous stirred tank reactors, CSTRs" nonlinear system is described in Figure 7.

The system has two identical series CSTR, and irreversible reactions take place inside the reaction tank. This system is usually used to increase the conversion rate of reactants. It is assumed that the reactions in both reactors take place under isothermal conditions, and the concentration of reactants in the reactors is controlled. The mathematical model of the two CSTR is obtained from the 

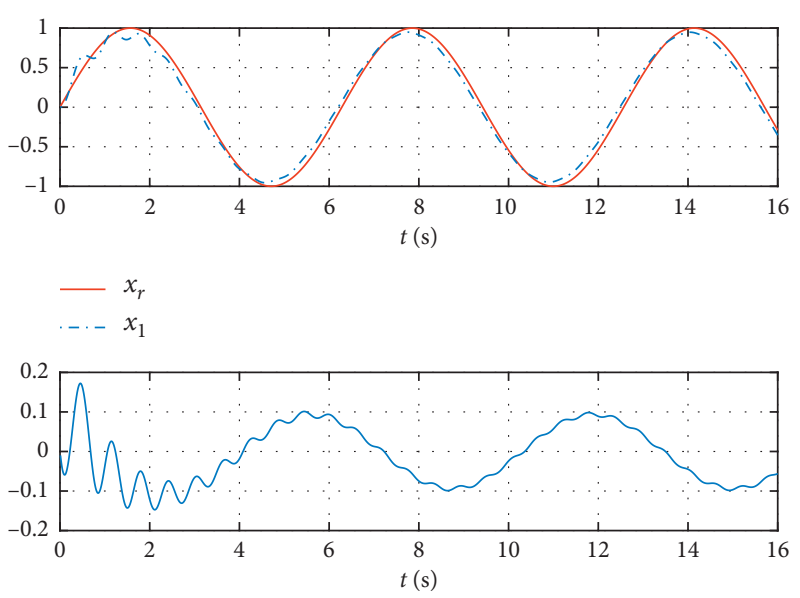

(a)

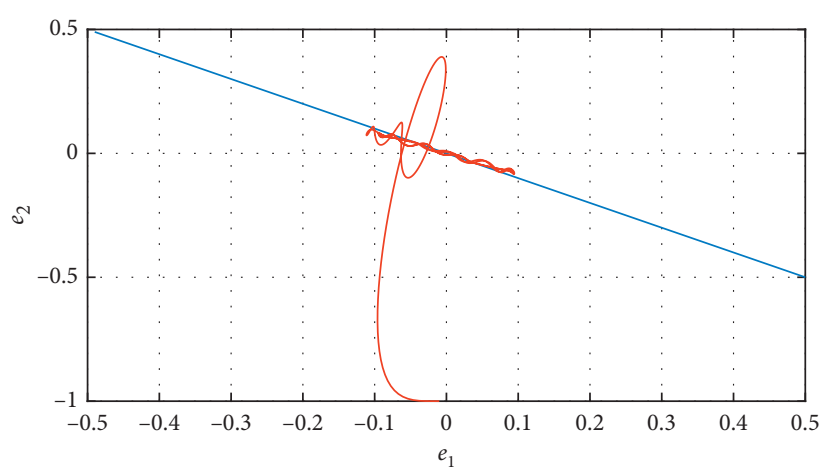

(b)

Figure 6: Simulation results in Example 1. (a) SMC tracking performance. (b) Sliding surface of Example 1.

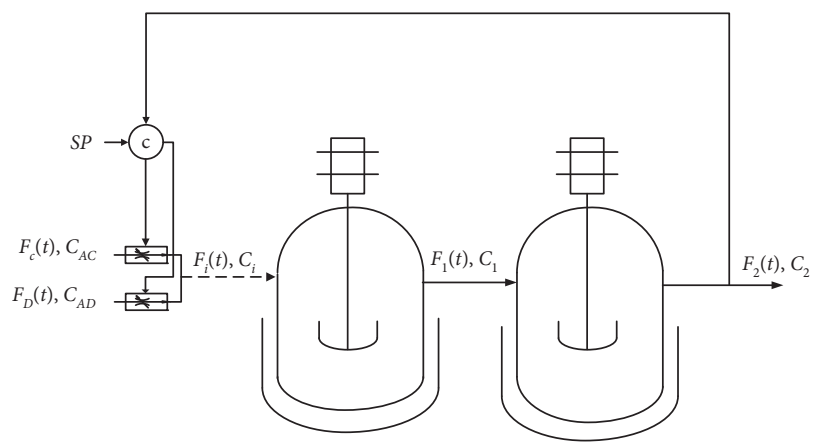

Figure 7: CSTRs.

law of mass conservation of reactants. The specific model is shown below:

$$
\begin{aligned}
& \left\{\begin{array}{l}
C_{A 2}(k+1)=\frac{F_{1} C_{A 1}(k)}{V_{2}}-\frac{F_{2} C_{A 2}(k)}{V_{2}}-K_{2} C_{A 2}^{j}(k), \\
C_{A 1}(k+1)=\frac{F_{i} C_{A i}(k)}{V_{1}}-\frac{F_{1} C_{A 1}(k)}{V_{1}}-K_{1} C_{A 1}^{j}(k), \\
y=C_{A 2}(k),
\end{array}\right. \\
& \left\{\begin{array}{l}
C_{A_{i}}(k)=\frac{F_{C}(k) C_{A C}+F_{D}(k) C_{A D}}{F_{C}(k)+F_{D}(k)}, \\
F_{D}(k)=F_{i},
\end{array}\right.
\end{aligned}
$$

with $K_{1}=\alpha_{1} e^{-(E / R T)}, K_{2}=\alpha_{2} e^{-(E / R T)}$ and variables with indices " 1 " and " 2 " refer to the first and second reactors. $C_{A}$ and $F$ are, respectively, concentration and flow rate of the reactants in the reactor, and $V$ denotes volume of reactors. $C_{A_{i}}$ and $F_{i}$ are, respectively, adjustable concentration and invariant reactant flow stream in the input. The chosen parameters for the simulation have been identified on CSTRs [29] and are presented in Table 2.

To simplify the system expression, let $x_{1}(k)=C_{A 2}(k), x_{2}(k)=C_{A 1}(k), u(k)=C_{A i}(k) ;(56)$ can be expressed as follows:

$$
\begin{aligned}
& \left\{\begin{array}{l}
x_{1}(k+1)=\frac{F_{1}}{V_{2}} x_{2}(k)+f_{1}\left(x_{1}(k)\right), \\
x_{2}(k+1)=\frac{1}{V_{1}} u(k)+f_{2}\left(x_{2}(k)\right), \\
y(k)=x_{1}(k),
\end{array}\right. \\
& \left\{\begin{array}{l}
f_{1}\left(x_{1}(k)\right)=-\frac{F_{2}}{V_{2}} x_{1}(k)-k_{2} x_{1}^{2}(k), \\
f_{2}\left(x_{2}(k)\right)=-\frac{F_{1}}{V_{1}} x_{2}(k)-k_{1} x_{2}^{2}(k),
\end{array}\right.
\end{aligned}
$$

in which the control input is constrained by saturation nonlinearity with parameters chosen as $\tau_{\min }=-1.8$, 
TABLE 2: CSTRs parameters.

\begin{tabular}{lcc}
\hline Parameter & Symbol & Value \\
\hline Inlet reactant feed stream & $F_{i}$ & $10(\mathrm{lit} / \mathrm{min})$ \\
The second reactor inlet flow stream & $F_{1}$ & $10(\mathrm{lit} / \mathrm{min})$ \\
The second reactor outlet flow stream & $F_{2}$ & $10(\mathrm{lit} / \mathrm{min})$ \\
The first reactor volume & $V_{1}$ & $25 \mathrm{lit}$ \\
The second reactor volume & $V_{2}$ & $25 \mathrm{lit}$ \\
The isothermal reaction coefficient 1 & $K_{1}$ & $0.428(\mathrm{lit} / \mathrm{mol} . \mathrm{min})$ \\
The isothermal reaction coefficient 2 & $K_{2}$ & $0.428(\mathrm{lit} / \mathrm{mol} . \mathrm{min})$ \\
The order of reaction & $j$ & 2 \\
Concentration of $A$ in diluted stream & $C_{A D}$ & $0(\mathrm{~mol} / \mathrm{lit})$ \\
Concentration of the concentrated stream & $C_{A C}$ & $20(\mathrm{~mol} / \mathrm{lit})$ \\
Energy of activation & $E$ & $6.46 \times 10^{4}(\mathrm{kl} / \mathrm{kmol})$ \\
The ideal gas constant & $R$ & $8.314(\mathrm{kl} / \mathrm{kmol} . \mathrm{C})$ \\
Temperature of the reactant & $T$ & $10^{0} \mathrm{C}$ \\
\hline
\end{tabular}

TABle 3: Value of coefficients in Example 2.

\begin{tabular}{lccccc}
\hline Parameter & $k_{1}$ & $k_{2}$ & $\delta$ & $\varrho$ & 0.01 \\
\hline Value & 7 & 5 & 0.25 & $h_{0}$ \\
Parameter & $\kappa_{1}$ & $\kappa_{2}$ & $\kappa_{3}$ & 0.01 \\
Value & 0.75 & $2 / 3$ & 0.5 & $1.5 * I_{25}$ & \\
\hline
\end{tabular}
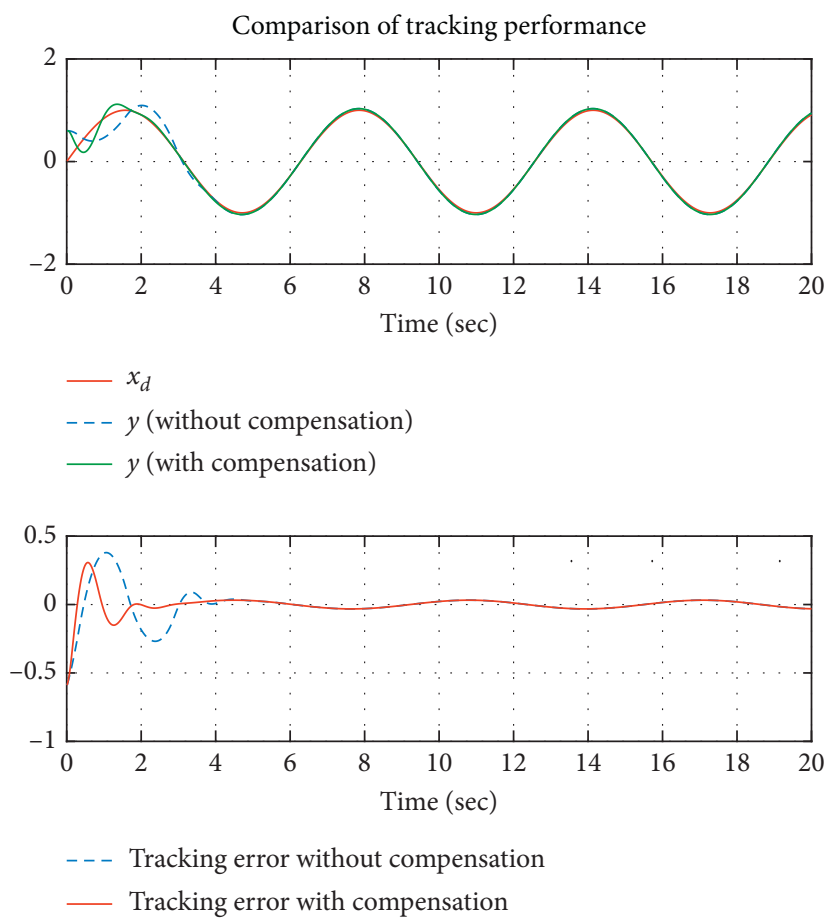

Figure 8: Tracking performance with/without compensation.

$\tau_{\max }=1.8, m=1$. The reference trajectory is $x_{r}=\sin (t)$. Based on the above CSTRs model, the proposed controller, differentiator, and observer are designed, and design parameter values are selected as shown in Table 3.

The simulation results to track a reference signal $x_{r}=$ $\sin (t)$ are depicted in Figures 8-12. Control performance and tracking errors are shown in Figure 8. Comparing the tracking errors with and without compensation in Figure 8, it can be seen that antiwindup control performance is achieved and tracking error is significantly reduced when applying the proposed fuzzy DSC control scheme. In addition, the curve of the control signal can be found to reduce the actuator power after compensation in Figure 9.

From the state observation curve in Figures 10 and 11, it can also be seen that unknown states are observed quickly and accurately by observer (11). From the analysis in Section 3 , it is concluded that better observation performance than HOSM observer and high gain observer (HGOB) can be 


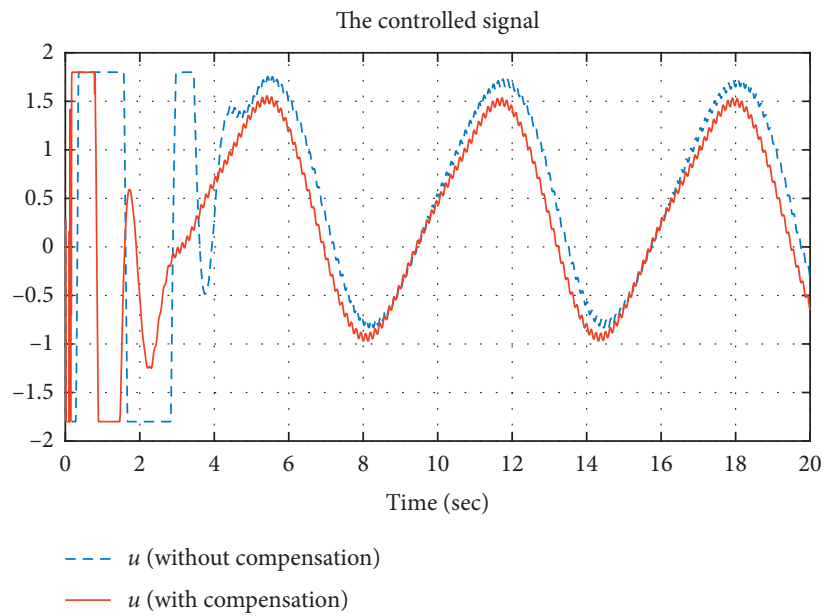

Figure 9: Control signal with/without compensation.
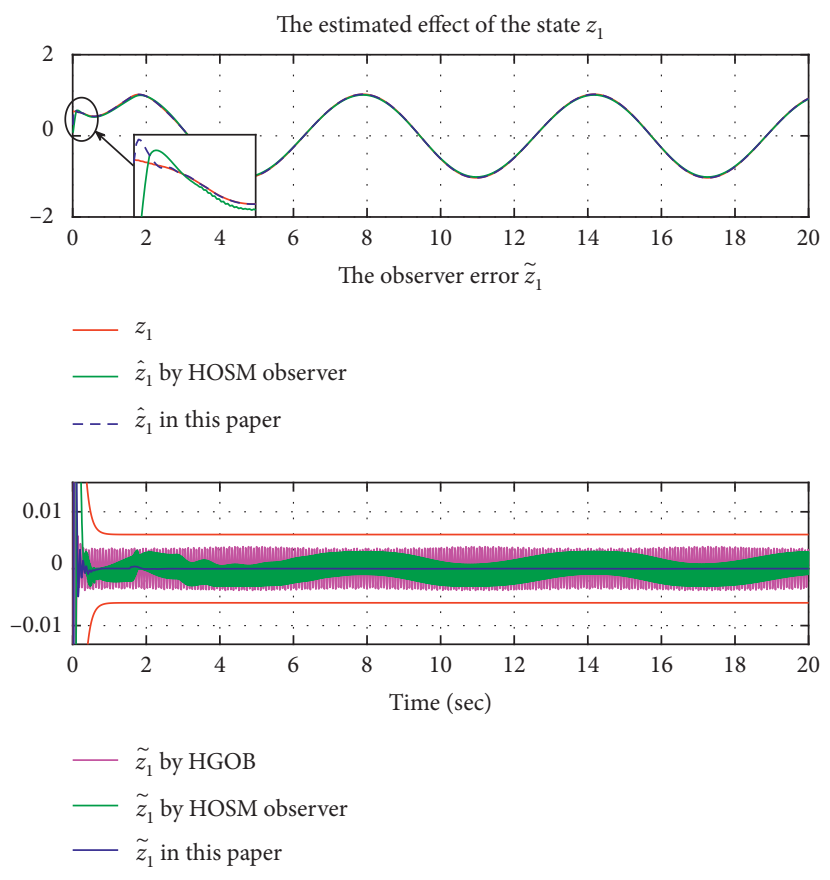

Figure 10: State $z_{1}$ of the observer. 

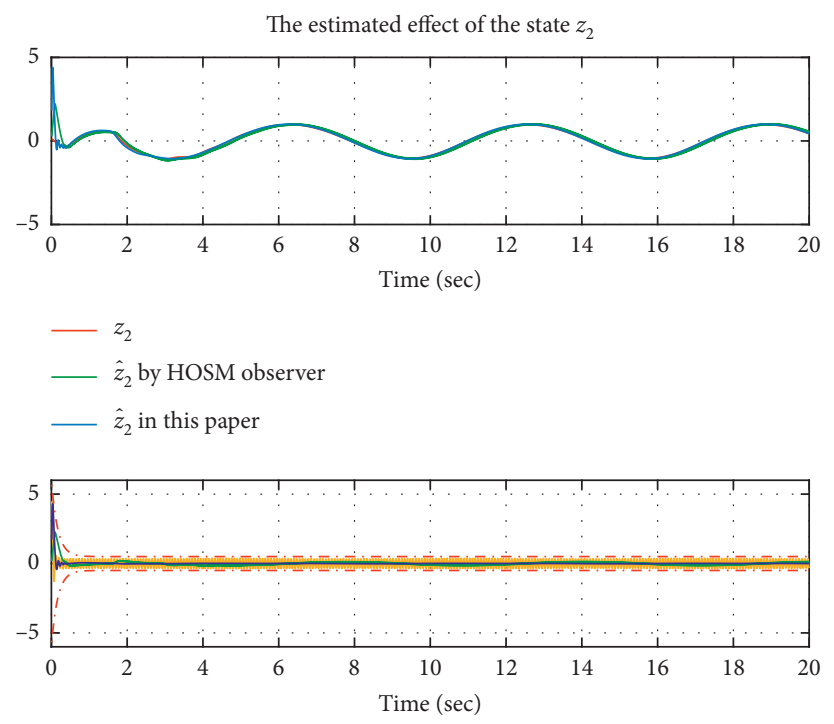

$\tilde{z}_{1}$ by HGOB
$\tilde{z}_{1}$ by HOSM observer
$-\tilde{z}_{1}$ in this paper

FIGURE 11: State $z_{2}$ of the observer.
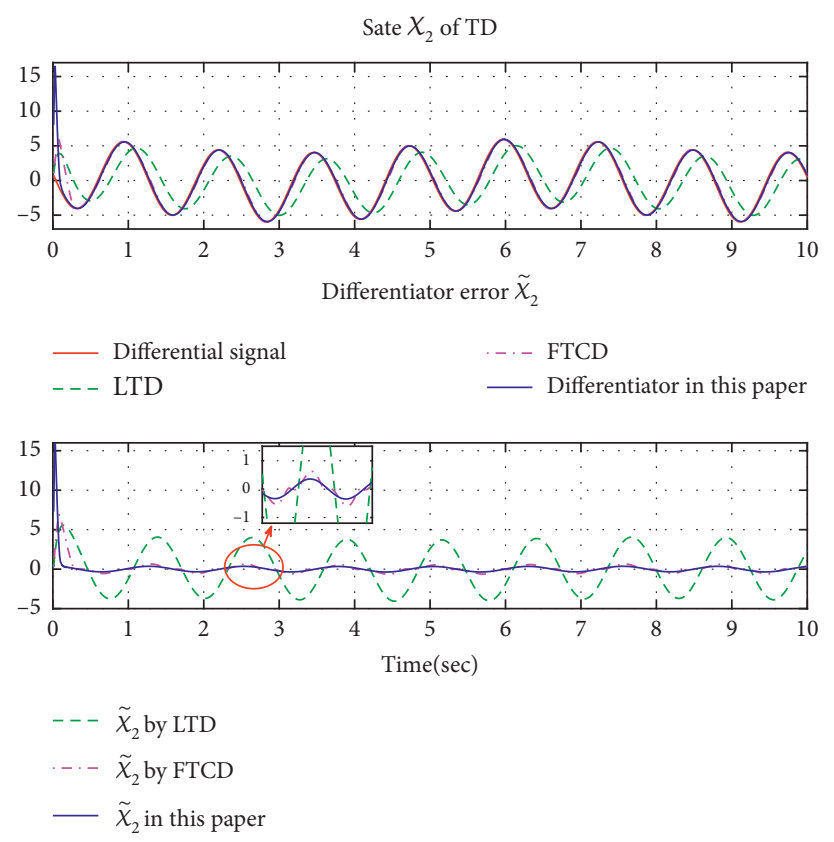

FIgURE 12: State $\chi_{2}$ of TD.

validated by curves of observation error in Figures 10 and 11. The design of observer (11) similarly makes the error convergent in finite time and effectively deals with the stable chattering problem of the HOSM observer.

In order to make a comparison effect, linear tracking differentiator (LTD) and finite-time convergent differentiator (FTCD) [30] were selected in the simulation and compared with the tracking differentiator (30) proposed in this paper. Figure 12 shows the comparison of differential signal tracking effects and errors of different differentiators. It can also be seen that nonlinear tracking differentiator used in this paper is obviously superior to linear tracking differentiator and error convergence speed is faster than that of FTCD. On the other hand, the buffeting problem is solved in the steady-state performance, so that the error curve that tends to be stable is more gentler. 
All these simulation results illustrate that the proposed fuzzy RDSC method effectively achieved the control objective proposed in Section 2.

\section{Conclusion}

In this paper, an adaptive fuzzy RDSC scheme that contains tracking differentiators and antiwindup fuzzy compensator is developed for improving the control performance of a class of nonlinear system with input saturation. Meanwhile, an improved HOSM observer is designed to achieve output feedback adaptive RDSC control. Specifically, only one FLS is employed to compensate for the total uncertainty in the last step of backstepping. Then, the stability and closed-loop performance are analyzed by Lyapunov theory. Finally, the tracking performance that guarantees the robustness of closed-loop system is validated by simulation examples.

\section{Data Availability}

The data used to support the findings of this study are available from the corresponding author upon request.

\section{Conflicts of Interest}

The authors declare that they have no conflicts of interest.

\section{Acknowledgments}

This work was supported by the National Natural Science Foundation of China under Grants 61703224 and 61620304.

\section{References}

[1] A. ur Rehman, M. Rehan, M. Riaz, M. Abid, and N. Iqbal, "Consensus tracking of nonlinear multi-agent systems under input saturation with applications: a sector-based approach," ISA Transactions, vol. 107, pp. 194-205, 2020.

[2] Z. Zuo, S. Guan, Y. Wang, and H. Li, "Dynamic event-triggered and self-triggered control for saturated systems with anti-windup compensation," Journal of the Franklin Institute, vol. 354, no. 17, pp. 7624-7642, 2017.

[3] Q. Zhou, L. Wang, C. Wu, H. Li, and H. Du, "Adaptive fuzzy control for nonstrict-feedback systems with input saturation and output constraint," IEEE Transactions on Systems, Man, and Cybernetics: Systems, vol. 47, no. 1, pp. 1-12, 2017.

[4] Y. Li, S. Tong, and T. Li, "Composite adaptive fuzzy output feedback control design for uncertain nonlinear strict-feedback systems with input saturation," IEEE Transactions on Cybernetics, vol. 45, no. 10, pp. 2299-2308, 2015.

[5] Y. Ouyang, L. Dong, Y. Wei, and C. Sun, "Neural network based tracking control for an elastic joint robot with input constraint via actor-critic design," Neurocomputing, vol. 409, pp. 286-295, 2020.

[6] L. Ren, C. Mao, Z. Song, and F. Liu, "Study on active disturbance rejection control with actuator saturation to reduce the load of a driving chain in wind turbines," Renewable Energy, vol. 133, pp. 268-274, 2019.

[7] M. R. Askari, M. Shahrokhi, M. Khajeh Talkhoncheh, and A. Moradvandi, "Observer-based adaptive fuzzy controller for uncertain non-strict state-delayed nonlinear systems subject to input and output constraints," Journal of the Franklin Institute, vol. 357, no. 12, pp. 7483-7514, 2020.

[8] Dinh, "Adaptive backlash compensation in upper limb soft wearable exoskeletons," Robotics and Autonomous Systems, vol. 92, pp. 173-186, 2017.

[9] M. C. Turner and M. Kerr, "A nonlinear modification for improving dynamic anti-windup compensation," European Journal of Control, vol. 41, pp. 44-52, 2018.

[10] S. Amini, B. Ahi, and M. Haeri, "Control of high order integrator chain systems subjected to disturbance and saturated control: a new adaptive scheme," Automatica, vol. 100, pp. 108-113, 2019.

[11] J.-J. Zhang, "Adaptive multi-dimensional taylor network dynamic surface control for a class of strict-feedback uncertain nonlinear systems with unmodeled dynamics and output constraint," ISA Transactions, vol. 108, pp. 35-47, 2021.

[12] C. Liu, C. L. P. Chen, Z. Zou, and T. Li, “Adaptive nn-dsc control design for path following of underactuated surface vessels with input saturation," Neurocomputing, vol. 267, pp. 466-474, 2017.

[13] L.-P. Xin, B. Yu, L. Zhao, and J. Yu, “Adaptive fuzzy backstepping control for a two continuous stirred tank reactors process based on dynamic surface control approach," Applied Mathematics and Computation, vol. 377, Article ID 125138, 2020.

[14] J. Yu, P. Shi, H. Yu, B. Chen, and C. Lin, "Approximationbased discrete-time adaptive position tracking control for interior permanent magnet synchronous motors," IEEE Transactions on Cybernetics, vol. 45, no. 7, pp. 1363-1371, 2015.

[15] S. Cong, L. Liu, D. Wang, Z. Peng, and Y. Zhang, "Modular neural dynamic surface control for position tracking of permanent magnet synchronous motor subject to unknown uncertainties," Neurocomputing, vol. 360, no. 7, pp. 163-171, 2019.

[16] S. B. Wang, J. Na, and Q. Chen, "Adaptive predefined performance sliding mode control of motor driving systems with disturbances," IEEE Transactions on Energy Conversion, vol. 11 page, 2020.

[17] Q. Chen, X. Yu, M. Sun, C. Wu, and Z. Fu, “Adaptive repetitive learning control of PMSM servo systems with bounded nonparametric uncertainties: theory and experiments," IEEE Transactions on Industrial Electronics, vol. 99, p. 1, 2020.

[18] YJ Liu, CL Chen, GX Wen, and S Tong, "Adaptive neural output feedback tracking control for a class of uncertain discrete-time nonlinear systems," IEEE Transactions on Neural Networks, vol. 22, no. 7, pp. 1162-1167, 2011.

[19] G. Zhang, J. Liu, Z. Liu, J. Yu, and Y. Ma, “Adaptive fuzzy discrete-time fault-tolerant control for permanent magnet synchronous motors based on dynamic surface technology," Neurocomputing, vol. 404, pp. 145-153, 2020.

[20] Y.-J. Liu and S. Tong, "Adaptive fuzzy control for a class of nonlinear discrete-time systems with backlash," IEEE Transactions on Fuzzy Systems, vol. 22, no. 5, pp. 1359-1365, 2014.

[21] L. Liu, Y. Wang, and Z. Gao, "Tracking control for the dynamic links of discrete-time complex dynamical network via state observer," Applied Mathematics and Computation, vol. 369, Article ID 124857, 2020.

[22] C. M. Agulhari and M. J. Lacerda, "Observer-based statefeedback control design for lpv periodic discrete-time systems," European Journal of Control, vol. 49, pp. 1-14, 2019. 
[23] D. Yang and X. Xie, "Observer-based state estimation of discrete-time nonlinear systems via a novel maximum-priority-based fuzzy observer," Signal Processing, vol. 137, pp. 63-68, 2017.

[24] E. Jafari and T. Binazadeh, "Observer-based tracker design for discrete-time descriptor systems with constrained inputs," Journal of Process Control, vol. 94, pp. 26-35, 2020.

[25] L. Moussaoui, S. Aouaouda, M. Chadli, O. Bouhali, and I. Righi, "State and output feedback control for constrained discrete-time nonlinear systems," European Journal of Control, vol. 50, pp. 79-87, 2019.

[26] JH Park, SH Kim, and CJ Moon, "Adaptive neural control for strict-feedback nonlinear systems without backstepping," IEEE Transactions on Neural Networks, vol. 20, no. 7, pp. 1204-9, 2009.

[27] Y.-J. Liu, L. Tang, S. Tong, and C. L. P. Chen, "Adaptive NN controller design for a class of nonlinear MIMO discrete-time systems," IEEE Transactions on Neural Networks and Learning Systems, vol. 26, no. 5, pp. 1007-1018, 2015.

[28] G. Zhiqiang, "On discrete time optimal control: a closed-form solution," in Proceeding of the 2004 American Control conference, pp. 52-58, Boston, MA, USA, June 2004.

[29] M. K. Talkhoncheh, M. Shahrokhi, and M. R. Askari, "Observer-based daptive neural network controller for uncertain nonlinear systems with unknown control directions subject to input time delay and saturation," Information Ences, vol. 27, no. 9, pp. 741-754, 2017.

[30] S. Yi, J. Wang, and B. Li, "Composite backstepping control with finite time convergence," Optik International Journal for Light and Electron Optics, vol. 142, 2017. 